\title{
Paraneoplastic Neurologic Syndrome: Three Case Reports
}

\author{
Emre ADIGÜZEL, Yasin DEMIR, Rıdvan ALACA, Arif Kenan TAN \\ Department of Physical Medicine and Rehabilitation, Gülhane Military Medical Faculty, Turkish Armed Forces Rehabilitation Center, Ankara, Turkey
}

\begin{abstract}
Paraneoplastic neurologic syndromes are a group of rare disorders that are triggered by an abnormal immune system response to a neoplasm. Paraneoplastic cerebellar degeneration, paraneoplastic limbic encephalitis and Guillain-Barre Syndrome are included by this group of disorders. In this case report, it was aimed to increase our knowledge about these rare syndromes by investigating three different patients with paraneoplastic neurologic syndrome after small cell lung cancer, immature teratoma and nodular sclerosing Hodgkin's lymphoma.
\end{abstract}

Keywords: Paraneoplastic neurologic syndrome, paraneoplastic cerebellar degeneration, limbic encephalitis, Guillain-Barre Syndrome

\section{Introduction}

Paraneoplastic neurological syndrome (PNS) is a rare neurological disorder that is triggered by an abnormal immune system reaction that develops as a response to malignancies. It often affects middle-aged and elderly people. It is mostly observed in patients with lung, ovary, lymphatic system, and breast cancers. Neurological symptoms usually develop before the diagnosis of tumor within days and weeks. The symptoms observed in these syndromes include gait disturbance, dysphagia, decreased muscle tone, fine motor coordination, decreased speech intelligibility, memory loss, visual problems, sensory deficits, dizziness, and epileptic seizures (1).

This study aims to present three different cases with PNS in order to emphasize the necessity of considering PNS in patients who have not yet been diagnosed despite having neurological symptoms and findings. The early detection of PNS can enable both early diagnosis and the treatment of malignancies and the early initiation of rehabilitation process.

\section{Case Reports}

\section{Case 1}

A 62-year-old male patient was admitted to our hospital with the complaints of weakness in the left lower extremity and speech disorder. It was learned from the medical history of the patient that his complaint of weakness had suddenly occurred, and speech disorder had developed after 2-3 days. The patient had a history of a transient ischemic attack that had healed without any sequela; he smoked two packs of cigarettes per day for 25 years. In the physical examination, he had atatic gait and speech. The muscle strengths of the dorsiflexor and plantar flexor in the left ankle were found to be $2 / 5$ in the muscle strength evaluation. His sensory examination was normal. A mild decrease was detected in the deep tendon reflexes of the left lower extremity. There was bilateral dysmetria. His cranial nerve examination was normal. In the laboratory examinations, complete blood count, routine biochemical tests, and urine test results were also normal. No pathology was detected in the cranial magnetic resonance imaging (MRI) and computed

Address for Correspondence: Emre Adıgüzel, MD, Gülhane Askeri Tıp Fakültesi, Fiziksel Tıp ve Rehabilitasyon Anabilim Dalı, Türk Silahlı Kuvvetleri Rehabilitasyon Merkezi, Ankara, Türkiye. Phone: +90 3122911401 E-mail: dremreadiguzel@gmail.com

Received: August 2013 Accepted: February 2014

OCopyright 2015 by Turkish Society of Physical Medicine and Rehabilitation - Available online at www.ftrdergisi.com

Cite this article as: 
tomography (CT) of the patient, and the examination of cerebrospinal fluid (CSF) was normal. No pathology of nerve root or second motor neuron involvement was found in the electrophysiological examination and MRI of the lumbar region, which were performed for determining the reason of muscle strength loss in the lower extremity. Severe vomiting and difficulty in swallowing were observed after that. The patient had no complaint regarding his respiratory system; however, the chest radiography, which was taken for control, revealed a $3 \times 5 \mathrm{~cm}$ mass in the right middle lobe. We suspected paraneoplastic cerebellar degeneration (PCD) in the case; because biopsy taken from the mass lesion revealed small cell lung cancer (SCLC), gait ataxia, dysarthria, and dysmetria were present and there was no other pathology which can explain this clinic. Anti-Yo test, which was performed for paraneoplastic antibody panel, was negative. Five chemotherapy sessions and 15 radiotherapy sessions were planned by the departments of medical oncology and radiation oncology clinics. During this period, the gait ataxia of the patient became more evident, his dysphagia increased, and percutaneous endoscopic gastrostomy was placed because of an aspiration that had developed. The patient, was also administered five plasmapheresis sessions, and he then underwent physical therapy and rehabilitation program. After the administration of plasmapheresis, healing was first observed in swallowing functions. The patient, who had short-distance ambulation with maximal support because of apparent ataxia before the physical therapy and rehabilitation program, started to walk long distance with a double tripod following balance, coordination, and gait exercises. Dysarthria was reduced with oro-motor strengthening exercises, and his speech became more understandable. In the control examination performed in the second month after the treatment program, it was observed that the patient could walk long distance with a double tripod, ataxic gait was almost completely improved, and his speech was more fluent and rapid.

\section{Case 2}

A 13-year-old female patient visited our hospital with the complaint of weakness in the left foot. It was learned from her history that her complaints had first started with mild amnesia, difficulty in focusing on something, and quickly getting tired. Approximately 2-3 months later, visual hallucinations, fever, headache, and crying attacks had suddenly occurred, and after one day, motor aphasia followed by nausea-vomiting, agitation, and inappropriate behaviors had developed in the patient. Immediately afterward, cardiac arrest had developed during a complex generalized seizure. The patient was admitted to the intensive care unit after resuscitation. In the chest radiography of the patient, a mass had been detected in the right lung, and a view that was consistent with limbic encephalitis had been observed in the brain MR imaging. In the immunological examination, anti-N-methyl D-aspartate (NMDA) receptor antibody had been found to be positive, and her she was diagnosed as anti-NMDA receptor-positive paraneoplastic limbic encephalitis (PLE). Viral encephalitis, metastasis, ischemic or hemorrhagic cerebrovascular diseases that could cause the existing findings, and toxic and metabolic pictures were ruled out on the basis of laboratory and imaging techniques. A $16 \times 14 \mathrm{~cm}$ mass in the right lung was excised by thoracic surgery department, and the pathological diagnosis was immature teratoma. Because of this diagnosis, were administered plasmapheresis and intravenous immunoglobulin (IVIG) treatments. Approximately 1 month after the mass excision, the patient begun to regain her consciousness. Her motor aphasia begun to resolve, and she could speak English and French, which she had previously known; furthermore, she started to speak Turkish in a few days. After her consciousness level had returned to normal, she was able to walk with small steps with the help of a walker, and thus, she was discharged. After discharge, she visited our hospital, and on examination, the strengths of her left knee flexors were $2 / 5$ and the strengths of the ankle dorsiflexor and plantar flexors were $1 / 5$. She had middle-distance ambulation, and her knee flexion during gait was insufficient. Electromyography examination revealed moderate-severe sciatic nerve lesion, and the patient used an ankle-foot orthosis (AFO). She underwent physical therapy and rehabilitation program. In the control examination that was performed after treatment, it was observed that the muscle strengths of the knee flexor and ankle plantar flexor and ankle dorsiflexor increased to $3 / 5$ and $2 / 5$, respectively. Furthermore, it was observed that she could perform heel strike while walking with an AFO, and she could independently walk long distance. Paraneoplastic neurological findings completely healed during the follow-up period; however, her isolated sciatic nerve lesion persisted, suggesting that the nerve lesion was probably associated with an intramuscular injection performed under intensive care conditions.

\section{Case 3}

A 53-year-old male patient was admitted to our clinic with the complaints of numbness and tingling sensation in the fingertips of both hands, which had started 4-5 days ago. According to his medical history, there was no specific finding except smoking for 35 years. In his physical examination, decreased deep tendon reflex in the upper extremity, mild bilateral dysdiadochokinesia, and dysmetria were detected. However, no pathological reflex was observed. His swallowing and speaking were normal. In the ultrasonographic examination that was performed because swelling was detected during the neck examination, multiple lymph nodes with echogenic hilus, the largest of which was $34 \times 17 \mathrm{~mm}$ in size, were observed adjacent to the right jugular vein in the infra-auricular region and supraclavicular posterior cervical region. The result of the first biopsy performed for the mass lesion was reported to be reactive lymphoid hyperplasia. Muscle strength loss in both lower and upper extremities, gait disturbance, and balance disorder were also added to the existent findings of the patient during this period. No pathological finding was found in his brain MRI. In the electromyographic examination, denervation potentials in the cervicothoracic and lumbosacral segments as well as mixed-type sensorimotor polyneuropathy were detected. In CSF evaluation, it was revealed that protein level apparently increased, and the cell count was normal. While our case was evaluated as a Guil- 
lain-Barre syndrome (GBS) on the basis of these findings, infectious pathologies that could lead to this clinical picture were ruled out during laboratory examination. After five IVIG therapy sessions administered by the neurology department, the complaint of gait disturbance was slightly reduced, and the patient could able to walk short-distance with a walker. Considering the etiology, biopsy was performed on the neck mass for the second time. After pathological examination, the diagnosis of nodular sclerosis Hodgkin lymphoma was established, and it was determined that GBS paraneoplastically occured. On the basis of this diagnosis, nine chemotherapy sessions and 15 radiotherapy sessions were administered to our patient, and then, physical therapy and rehabilitation program was initiated. The patient used a walker to ambulate long distance with the help of walking, balance, and strengthening exercises. His sitting balance improved from moderate to good level.

\section{Discussion}

PNS which can affect some areas of the nervous system from the cerebral cortex to the neuromuscular junction and the muscle, is a rare clinic. However, it is important because it appears before cancer is diagnosed or when cancer is very small and can be treated. While neurological symptoms develop as the first signs of tumor in $70 \%$ of PNS cases (2), and tumor can be detected in approximately $70 \%-80 \%$ of these cases during the initial evaluation (3).

Today, it is believed that most PNS occur because of the immune response that develops against neural proteins expressed by a tumor (4). The detection of some antibodies in CSF evaluations of patients supports immune-mediated pathogenesis (5). Furthermore, PNS can be caused by some other non-immune reasons apart from this mechanism. The formation of metabolic abnormalities through hormones and/or cytokines released from a tumor (antidiuretic hormone-induced hyponatremia, hypoglycemia associated with insulin-like growth factor, etc.), the competition between a tumor and the nervous system for a specific substrate (tryptophan in carcinoid tumors), and the effect of immunoglobulins released from a tumor on the peripheral nervous system (such as sensorimotor peripheral neuropathy in Waldenstrom macroglobulinemia) are among these reasons (2).

Autoantibodies that occur in PNS develop against the surface antigens or intracellular components of the nervous system cells. These antibodies are divided into two groups: well characterized and partially characterized. While well-characterized paraneoplastic antibodies are definitely associated with PNS and they are defined in many cases by a lot of researchers, partially characterized antibodies, the definite target of which is unknown, are demonstrated in a few cases (4).

PCD is among the most frequent paraneoplastic syndromes. They often appear with gynecological cancers, breast cancer, SCLC, and Hodgkin lymphoma $(6,7)$. Before neurological deficits, prodromal complaints, such as dizziness, nausea, vomiting, and flu-like symptoms, can be observed (8). Following these symptoms, ataxic gait, dysarthria, dysphagia, and diplopia can develop. Blurred vision, oscillopsia, and transient opsoclonus can appear in some patients $(7,9,10)$. The result of MRI in early stage is often normal in most patients. While transient diffuse cerebellar expansion or increased cortico-meningeal distance can develop in some patients (11), cerebellar atrophy can be observed in MRI over time.

Antibodies related to cerebellar degeneration include AntiYo, Anti-Tr, Anti-Hu, Anti-Ma2, and Anti-Ri (5). Among these antibodies, Anti-Yo (Purkinje cell antibody type-1) and Anti-Tr are well-characterized antibodies for PSD.

The sudden onset of cerebellar dysfunction symptoms, history of smoking, the absence of apparent risk factors except transient ischemic attack, diagnosis of SCLC, and absence of any other clinical picture that could explain the clinical signs and findings after all examinations, including cranial MRI and $C T$, suggested the diagnosis of PCD in our first case. Because the diagnosis of cancer accompanying classical syndrome findings meets the definite PCD criteria (4), negative Anti-Yo antibody did not rule out the diagnosis of PNS in our case. As in our case, when symptoms and findings related to cerebellar activation are encountered during rehabilitation practice, central nervous system metastases, toxic and metabolic causes, alcoholism, and vitamin deficiency should also be remembered.

Because PCD clinically affects the functional state of patient notably, comprehensive rehabilitation procedures, including speaking, swallowing, and occupational therapies, are required. During this period, attention should be paid to aspiration risk, appropriate nutrition method should be chosen, and gastrostomy should be used to provide nutrition when necessary (12).

Immunotherapy is frequently used in treatments, but its remarkable effects on the results of a disease should be discussed. In the PSD case series of Shams'ili et al. (6), it was reported that immunotherapy and anti-tumor treatments did not affect the functional results of the disease. However, there are some studies reporting that immunotherapy is effective $(13,14)$. In our case, it was revealed that chemotherapy, radiotherapy, and immunotherapy before the rehabilitation process provided positive contributions to the functional results of the patient.

Prominent findings of paraneoplastic and autoimmune limbic encephalitis are recent memory loss, changes in behaviors and mood, and epileptic seizures $(15,16)$. In addition to these findings, hyperthermia due to hypothalamic dysfunction, somnolence, and endocrine disorders can also develop. Although it has been reported in many case series that PLE mostly affects adult patients, there are also some studies demonstrating that adolescents and children can also undergo limbic encephalitis (17). The study conducted by Haberlandt et al. (17) with 10 patients with PLE, whose ages varied between 3 and 17 years and who were from three different centers in Europe, and our second case, who was 13 years old, support that PLE can also develop in early ages.

PLE is often associated with lung (SCLC), seminoma, and other testicular tumors, thymoma, Hodgkin lymphoma, or ovarian teratomas (15). The development of PLE because of mediastinal teratoma is rare. Two case reports on paraneoplastic encephalitis secondary to mature mediastinal teratoma, which were conducted by Kawahara et al. (18) and Strover et al. (19), are available. In our case, the development of PLE because of 
immature mediastinal teratoma is remarkable.

The demonstration of epileptic activity in electroencephalographic examination and unilateral or bilateral temporal lobe activation in MR, CSF, and autoantibody examinations are necessary for the diagnosis of PLE. In the evaluation of the clinical picture of the second case, it is noticed that the diagnosis of cancer was rapidly established in the patient and the diagnosis of PLE was supported by increased bilateral hippocampal signal and anti-NMDA positivity. In the second case, tumor excision surgery performed at early stage, plasmapheresis, and IVIG therapies enabled the early initiation of the rehabilitation process and contributed more to the quality of life compared with the other two cases. Although epileptic seizures occurred in the acute periods of the disease in these cases, it must be kept in mind that they can develop during rehabilitation process, and thus, must be treated with antiepileptic drugs (20).

GBS includes an acute immune-mediated polyneuropathies that often appears following an infection. It is considered that both humoral and cellular immunity play a role in the development of the disease $(21,22)$. GBS mostly develops secondary to the respiratory and gastrointestinal system infections or vaccination. However, there are also case reports demonstrating that it accompanies cancer $(23,24)$. While the cancer type that is associated to GBS more frequently is Hodgkin lymphoma (25, $26)$, its relationship with other cancer types is less known. Some researchers emphasize that the development of GBS with cancer is coincidental (27); however, in a study conducted by Vigliani et al. (28), it was reported that cancer concurrently developed with GBS or it developed following GBS in nine of 435 patients with GBS, and it was stated that this relationship could not be coincidental. Clinical findings in paraneoplastic GBS are not different from those in post-infectious GBS. Of patients, $80 \%$ can walk in the first 6 months and $84 \%$ can independently walk within 1 year. Approximately 5\%-10\% can remain dependent on a ventilator, and late or incomplete recovery can be observed (29). In $10 \%$ of cases, while GBS can demonstrate relapses, plasma change applied in relapse treatment or the response of patient to IVIG therapy can be partial or complete. In contrast, in $2 \%$ cases, recurrent muscle strength loss can be observed in association with the development of chronic inflammatory demyelinating polyradiculoneuropathy (30). As in our third case, the response of the patient to IVIG therapy was incomplete. It is believed that some other factors, such as the patient not having got rid of the tumor because the mass associated with Hodgkin lymphoma was in an inoperative stage, the antigenic source not having been completely eliminated, and the patient having undergone only chemotherapy and radiotherapy, affected paraneoplastic GBS remission. Complications, such as deep vein thrombosis, autonomic dysfunction, respiratory failure, immobilization hypocalcaemia, and decubitis, can develop particularly in acute stages of GBS $(31,32)$. During the rehabilitation of our case, the complications stated above were not observed, and the rehabilitation process was not negatively affected.

\section{Conclusion}

If etiological diagnosis is unclear in patients presenting with different neurological symptoms and findings, it should be considered that this clinical picture can be associated with PNS. It should be kept in mind that neurological findings can occur many months, even years, before the diagnosis of cancer. Furthermore, it should be understood that early detection of PNS is important for the early treatment of tumor and early initiation of rehabilitation through rapid diagnosis of tumor.

Informed Consent: Written informed consent was obtained from patients.

Peer-review: Externally peer-reviewed.

Author Contributions: Concept - R.A., E.A.; Design - E.A., R.A.; Supervision - R.A., A.K.T.; Resource - E.A., R.A.; Materials - R.A., E.A.; Data Collection and/or Processing - E.A., Y.D.; Analysis and/or Interpretation - E.A., R.A.; Literature Review - E.A., Y.D.; Writer - E.A., Y.D.; Critical Review - R.A., A.K.T.

Conflict of Interest: No conflict of interest was declared by the authors.

Financial Disclosure: The authors declared that this study has received no financial support.

\section{References}

1. National Institute of Neurological Disorders and Stroke Paraneoplastic Syndromes Information Page. Available from: URL: http:// www.ninds.nih.gov/disorders/paraneoplastic/paraneoplastic.htm. Accessed June 12, 2013.

2. Posner JB. Paraneoplastic syndromes. In: Posner JB, editor. Neurologic Complications of Cancer. Philadelphia: FA Davis; 1995. p.35385.

3. Younes-Mhenni S, Janier MF, Cinotti L, Antoine JC, Tronc F, Cottin V, et al. FDG-PET improves improves tumor detection in patients with paraneoplastic neurological syndromes. Brain 2004;127:2331-8. [CrossRef]

4. Graus F, Delattre JY, Antoine JC, Dalmau J, Giometto B, Grisold W, et al. Recommended diagnostic criteria for paraneoplastic neurological syndromes. J Neurol Neurosurg Psychiatry 2004;75:1135-40. [CrossRef]

5. Dalmau J, Rosenfeld MR. Paraneoplastic syndromes of the CNS. Lancet Neurol 2008;7:327-40. [CrossRef]

6. Shams'ili S, Grefkens J, de Leeuw B, van den Bent M, Hooijkaas $H$, van der Holt $\mathrm{B}$, et al. Paraneoplastic cerebellar degeneration associated with antineuronal antibodies: analysis of 50 patients. Brain 2003;126:1409-18. [CrossRef]

7. Peterson K, Rosenblum MK, Kotanides H, Posner JB. Paraneoplastic cerebellar degeneration. I. A clinical analysis of 55 anti-yo antibodypositive patients. Neurology 1992;42:1931-7. [CrossRef]

8. Dalmau J, Gonzalez RG, Lerwill MF. Case records of the Massachusetts General Hospital. A 56-year-old woman with rapidly progressive vertigo and ataxia. N Engl J Med 2007;356:612-20. [CrossRef]

9. Rosenfeld MR, Eichen JG, Wade DF, Posner JB, Dalmau J. Molecular and clinical diversity in paraneoplastic immunity to Ma proteins. Ann Neurol 2001;50:339-48. [CrossRef]

10. Yu Z, Kryzer TJ, Griesmann GE, Kim K, Benarroch EE, Lennon VA. CRMP-5 neuronal autoantibody: marker of lung cancer and thymoma-related autoimmunity. Ann Neurol 2001;49:146-54. [CrossRef]

11. de Andrés C, Esquivel A, de Villoria JG, Graus F, Sánchez-Ramón S. Unusual magnetic resonance imaging and cerebrospinal fluid findings in paraneoplastic cerebellar degeneration: a sequential study. J Neurol Neurosurg Psychiatry 2006;77:562-3. [CrossRef] 
12. Lin JT, Lachmann E, Nagler W. Paraneoplastic cerebellar degeneration as the first manifestation of cancer. J Womens Health Gend Based Med 2001;10:495-502. [CrossRef]

13. David YB, Warner E, Levitan M, Sutton DM, Malkin MG, Dalmau JO. Autoimmune paraneoplastic cerebellar degeneration in ovarian carcinoma patients treated with plasmapheresis and immunoglobulin. A case report. Cancer 1996;78:2153-6. [CrossRef]

14. Blaes F, Strittmatter M, Merkelbach S, Jost V, Klotz M, Schimrigk K, et al. Intravenous immunoglobulins in the therapy of paraneoplastic neurological disorders. J Neurol 1999;246:299-303. [CrossRef]

15. Gultekin SH, Rosenfeld MR, Voltz R, Eichen J, Posner JB, Dalmau J. Paraneoplastic limbic encephalitis: neurological symptoms, immunological findings and tumour association in 50 patients. Brain 2000;123:1481-94. [CrossRef]

16. Tüzün E, Dalmau J. Limbic encephalitis and variants: classification, diagnosis and treatment. Neurologist 2007;13:261-71. [CrossRef]

17. Haberlandt $E$, Bast $T$, Ebner A, Holthausen $H$, Kluger $G$, Kravljanac $\mathrm{R}$, et al. Limbic encephalitis in children and adolescents. Arch Dis Child 2011;96:186-91. [CrossRef]

18. Kawahara K, Miyawaki M, Anami K, Moroga T, Yamamoto S, Tokuishi $\mathrm{K}$, et al. A patient with mediastinal mature teratoma presenting with paraneoplastic limbic encephalitis. J Thorac Oncol 2012;7:258-9. [CrossRef]

19. Strover DG, Eisenberg R, Johnson DH. Anti-n-methyl-d-aspartate receptor encephalitis in a young woman with a mature mediastinal teratoma. J Thorac Oncol 2010;5:1872-3. [CrossRef]

20. Dalmau J, Lancaster E, Martinez-Hernandez E, Rosenfeld MR, Balice-Gordon R. Clinical experience and laboratory investigations in patients with anti-NMDAR encephalitis. Lancet Neurol 2011;10:6374. [CrossRef]

21. Hartung HP, Pollard JD, Harvey GK, Toyka KV. Immunopathogenesis and treatment of the Guillain-Barré syndrome -- Part I. Muscle Nerve $1995 ; 18: 137-53$. [CrossRef]
22. Vedeler CA. Inflammatory neuropathy: update. Curr Opin Neurol 2000;13:305-9. [CrossRef]

23. Croft $\mathrm{PB}$, Urich $\mathrm{H}$, Wilkinson $\mathrm{M}$. Peripheral neuropathy of sensorimotor type associated with malignant disease. Brain 1967;90:31-66. [CrossRef]

24. Rodrigues A, Monteiro A,Viana J,Macedo A, Graca F, Sena A. Acute non-lymphoblastic leukemia presenting as a Guillain-Barré syndrome. J Neurol Neurosurg Psychiatry 1993;56:936-7. [CrossRef]

25. Lunn MP, Nobile-Orazio E. Immunotherapy for IgM anti-Myelin-Associated Glycoprotein paraprotein-associated peripheral neuropathies. Cochrane Database Syst Rev 2003:CD002827. [CrossRef]

26. Julien J, Vital C, Aupy G, Lagueny A, Darriet D, Brechenmacher C. Guillain-Barré syndrome and Hodgkin's disease--ultrastructural study of a peripheral nerve. J Neurol Sci 1980;45:23-7. [CrossRef]

27. Ropper AH, Wijdicks EFM, Truax BT. Guillain-Barré syndrome. Philadelphia: F.A.Davis; 1991.

28. Vigliani MC, Magistrello M, Polo P, Mutani R, Chio A; Piemonte and Valle d'Aosta Register for Guillain-Barré Syndrome. Risk of cancer in patients with Guillain-Barre syndrome (GBS). A population based study. J Neurol 2004;251:321-6. [CrossRef]

29. Kissel JT, Cornblath DR, Mendell JR. Guillain-Barre syndrome. In: Mendell JR, Kissel JT, Cornblath DR, editors. Diagnosis and Management of Peripheral Nerve Disorders. New York: Oxford University Press; 2001. p.145-72.

30. Odaka M, Yuki N, Hirata K. Patients with chronic inflammatory demyelinating polyneuropathy initially diagnosed as Guillain-Barré syndrome. J Neurol 2003;250:913-6. [CrossRef]

31. Meythaler JM. Rehabilitation of Guillain-Barré syndrome. Arch Phys Med Rehabil 1997;78:872-9. [CrossRef]

32. Hughes RA, Wijdicks EF, Benson E, Cornblath DR, Hahn AF, Meythaler JM, et al. Supportive care for patients with Guillain-Barré syndrome. Arch Neurol 2005;62:1194-8.[CrossRef] 